\title{
Optimal Scheduling for Networks of RFID Readers
}

\author{
Vinay Deolalikar, John Recker, Malena Mesarina, and Salil Pradhan \\ Hewlett-Packard Labs, Palo Alto CA 94304 \\ vinayd@hpl.hp.com
}

\begin{abstract}
Devising switching schemes for networks of colliding and correlated RFID readers is a core challenge in the deployment of RFID networks. We derive optimal scheduling schemes for readers in RFID networks in four cases of practical importance. Most other cases can be reduced to a combination of these basic cases.
\end{abstract}

\section{Introduction}

RFID (Radio Frequency Identification) technology is expected to play a central role in asset tracking and management in the near future. RFID networks are being deployed to track the flow of assets through various environments. The central question concerning the efficient functioning of such networks may now be posed as follows. Given the topology of the RFID network, including information on potential collisions between readers, and the correlations between the various RFID readers, what is the optimum scheduling of the RFID readers in the network? Indeed, an answer to this question must lie at the heart of every efficient algorithm to schedule readers in a RFID network. This article answers this question in several scenarios that commonly occur in practical deployments of RFID systems.

Our work seeks to combine approaches and insights from two different areas. First, there is the literature from the collaborative signal and information processing (CSIP) community, such as [6], [7]. However, in the scenarios we examine in this paper, communication and processing resources are not primary bottlenecks. However the end goal of an RFID system is the same: to maximize the amount of information extracted from the RFID tags in the environment.

The second area from which we extract techniques started with the application of graph theoretic tools to the problem of assigning radio frequency spectrum to a set of radio frequency transmitters, such as cellular telephone base stations [4] 5. This work was latter re-examined in the context of frequency assignment in multi-channel (multi-frequency) RFID reader systems in 2. Thus graphical models found their way into the analysis of RFID reader collision problems. This effort has been extended in another paper by the authors where they consider perturbations of these graphical models [1].

Consequently, the methods of this paper are derived from information processing as well as graph theory.

\footnotetext{
^ Corresponding author.
} 


\section{RFID Reader Scheduling}

Reader Model: In this paper, an RFID reader reading a fixed set of tags is modeled as operating in two time regions, an acquisition region, and a saturation region as shown in figure 1 (a). In the acquisition region, a reader has less than the minimum amount of time $T_{s}$ necessary for it to read its maximum number of tags. We assume that in the acquisition region, the number of tags read increase linearly with time, till they reach a maximum at time $T_{s} . T_{s}$ is referred to as the saturation time of the reader. In the saturation region, the reader has more than the time $T_{s}$ required to read its maximum number of tags.

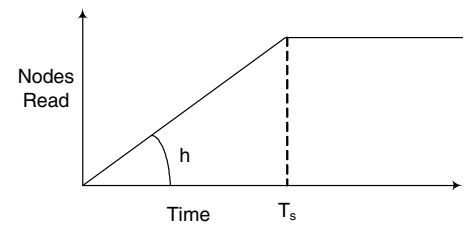

(a)

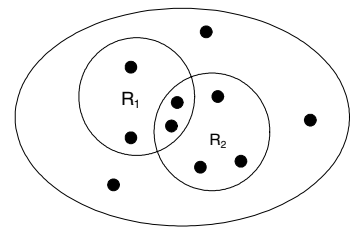

(b)

Fig. 1. a) Number of tags read by RFID reader with time. b) RFID reader correlation. $\operatorname{Corr}_{R_{1}}=\frac{4}{10}=0.4, \operatorname{Corr}_{R_{2}}=0.5$, Cross correlation $\operatorname{Corr}_{1}^{2}=R_{1} \cap R_{2}=0.2$.

Remark 1. While we assume a linear approximation to the acquisition region, it can be an arbitrary well behaved function during that time interval and yet be amenable to our method of analysis.

We can now formally state our goal. Let $T$ be the time period of operation of the RFID network and $T_{i}$ be the time allocated to the $i^{\text {th }}$ reader. Then our goal is to maximize the number of distinct tags read under the constraint $\sum_{i} T_{i}=T$.

To achieve this goal, we start with an elementary, but useful, observation. There is no advantage to giving more time than $T_{s_{i}}$ to the $i^{\text {th }}$ reader, since beyond $T_{s_{i}}$ it does not read any more tags. It follows that we should operate all readers in their acquisition region. Therefore we impose the constraint $T_{i} \leq T_{s_{i}}$. If we assume a linear tag read rate in the acquisition region for all the RFID readers in the network, with a slope of $h_{i}$ for the $i^{t h}$ reader, the network will read at most $N$ tags, where $N \leq \sum_{i} h_{i} T_{i}$. Equality is achieved when all reads are disjoint.

In the following sections, we will find optimal switching schemes for various simple topologies of RFID readers and tags. We assume that tags are contained inside boxes, which are passing through the RFID reader system. Throughout the rest of this article, we will assume that the following information is either available or the means to compute it are available to us.

1. The interrogation zone overlap for each pair of readers,

2. Each reader's rate of tag read, and

3. Statistical correlation between each reader's total tags read at saturation and total tags in a typical box. 


\section{Correlation and Normalization}

The use of correlation information is central to our analysis. First we explain what correlation means in the context of RFID readers and how we intend to compute it's numerical value.

There are two correlations that we need. The first is the correlation between a reader and the event, which in our case is a box of tags. We model this simply as the fraction of tags in the box that are correctly read by the reader. Cross correlation between two readers is modeled similarly. It is the fraction of tags that are read by both the readers. We can extend this to correlation between any number of readers similarly (see figure 1 (b) ).

In practice, what we would do is to pass many boxes through the RFID reader network, and note these fractions for each box and then average out so as to get a statistical correlation that reflects the particular topology and environment of deployment of the network.

One thing that cross-correlation allows us to do is to "normalize" the correlation of a reader to reflect the number of unique tags a reader will read from the remaining unread tags in a previously scanned stream of tags. We formalize this notion below.

Definition 1. The normalized correlation of the $i^{\text {th }}$ RFID reader with respect to a set of readers $S=\left\{R_{j}\right\}_{j \neq i}$ is a scaling of the original correlation Corr $r_{i}$ by its cross-correlation with each of the RFID readers in $S$. Thus the normalized correlation of the $i^{\text {th }}$ reader with respect to $S$ is given by

$$
\operatorname{Corr}_{i}^{S}=\operatorname{Corr}_{i} \prod_{R_{j} \in S}\left(1-r_{i j}\right)
$$

where $r_{i j}$ is the cross-correlation between the $R_{i}$ and $R_{j}$.

Remark 2. Note that in the definition above, the normalized correlation refers to the correlation between a reader and the event.

Definition 2. A virtual reader is a reader whose correlation with an event has been normalized with respect to a fixed set of readers.

Remark 3. The process of creating virtual readers does not change the saturation time $T_{s}$ of the reader. Though some reads from virtual readers will prove to be redundant, each reader must still read its full complement of tags.

The use of correlation allows us to work around two important problems. First, as noted above, our goal is to maximize the number of distinct tags read. Correlation allows us to approach this problem statistically by keeping account of the number of tags that are read in common by sets of readers.

Next, RFID reader system can often be quite complex, with many readers, overlapping interrogation zones, and different path lengths of the tags within each reader's interrogation zone. Furthermore, the physical characteristics of the readers, box, conveyor belt and tags are highly system dependant. Correlation 
allows us to summarize all the system dependant characteristics of the readers in a single number. Furthermore, this number can be determined experimentally for each subset of readers in a system as explained earlier.

\section{One Box, Multiple Readers}

We start by examining the scenario depicted in figure 2 A single box with a number of RFID tags moves along a conveyor belt, through the interrogation zones of several RFID readers. These interrogation zones overlap as shown in the figure. Although the figure shows only three readers, we will see that the algorithm we will provide works for any number of readers.

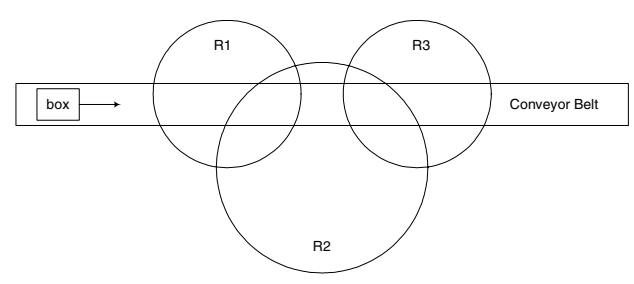

Fig. 2. Single box passing by several RFID readers

Due to the overlaps, operating reader $R_{2}$ simultaneously with either $R_{1}$ or $R_{3}$ will result in a collision and therefore no reads for either of the readers. This rules out the stratagem of keeping all the three readers switched on at all times and simply collating their reads. The objective then is to find the optimal switching scheme for this topology of readers and tags.

Recalling our reader model where the number of tags read is linearly proportional to the reading time up to the saturation time, the total number of tags read is then given by

$$
N=\sum_{i} h_{i} T_{i}, \quad T_{i} \leq T_{S_{i}} .
$$

The constraint on time is

$$
T=\sum_{i} T_{i} \leq \sum T_{s_{i}}
$$

Equation 2 is the equation of a hyper-plane. And the objective is to find the maximum of this hyperplane function under the constraints given by Equation 3 . These constraints define the portion of the hyperplane that lies entirely in the positive quadrant, as shown in Figure 3 (a).

The candidates for the maximum value of this function must lie on the boundary, and indeed must be one of the three intercepts with the axes. The largest intercept corresponds to the reader with the highest read rate. Of course, this is true only so far as that reader saturates. 


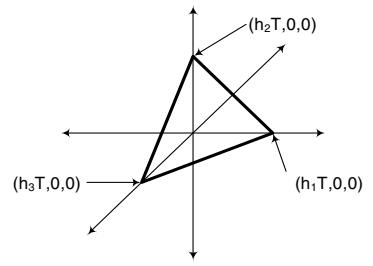

(a)

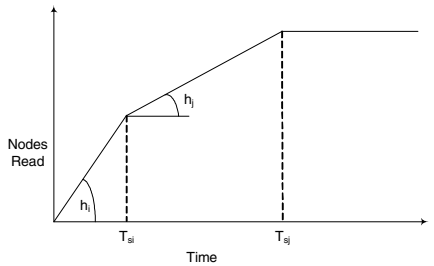

(b)

Fig. 3. Alternate visualizations of equation 2 a) Hyper-plane for 3 RFID readers. b) Summing contributions from successive readers.

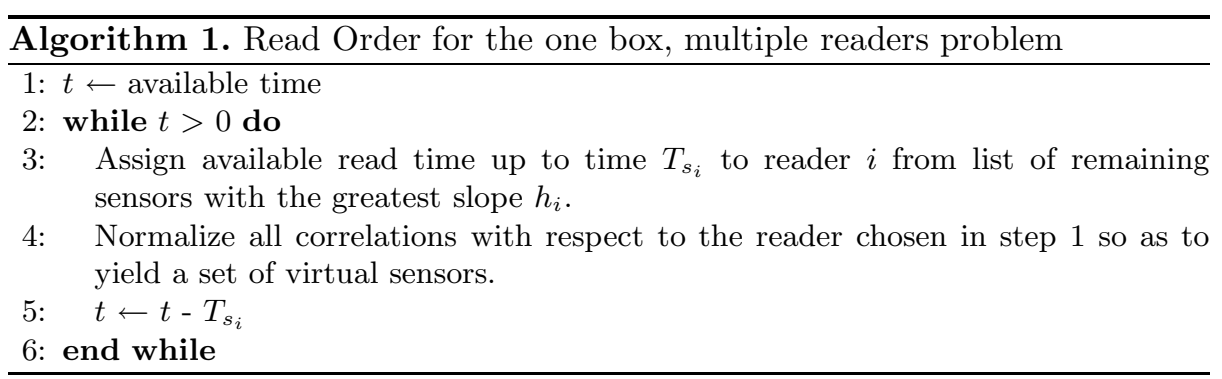

The algorithm for assigning read order and time thus proceeds as follows.

Note that there is no need to extend step 1 in the algorithm by firing other readers that do not collide with reader $R_{i}$. This is because there is only one box in the system, and so if it is in the field of $R_{i}$, it cannot possibly be in the field of a reader that does not collide with $R_{i}$, yielding no benefit to firing that reader. This is clearly not the case for multiple boxes.

\section{Continuous Box Stream, One Reader}

Section 4 solved the problem of a single box moving past a collection of RFID readers, some of whose fields overlapped. We will now examine the case of a continuous stream of boxes moving with velocity $d$ and tag density $d$ through the interrogation field of a RFID reader $R_{1}$, as shown in figure 4 (a). It is obvious that that optimum strategy is to have reader $R_{1}$ reading at all times. However, we wish to derive an expression for the number of tags a typical reader can be expected to read.

We first make the observation that for a given tag in a differential region $d x$ situated $x$ units into its field, the probability that the reader $R_{1}$ has read the tag is

$$
P_{d x}(\text { Read })=\left[\frac{\frac{x}{v}}{T_{s_{1}}}\right] \operatorname{Corr}_{R_{1}} .
$$

It follows that the average number of tags read in the differential region $d x$, denoted by TagsRead ${ }_{d x}$, is 


$$
\operatorname{TagsRead}_{d x}=P_{d x}(\operatorname{Read})(d x * d)=\left[\frac{\frac{x}{v}}{T_{s_{1}}}\right] \operatorname{Corr}_{R_{1}}(d x * d) .
$$

The total number of tags read on the average, denoted TagsRead, therefore is

$$
\text { TagsRead }=\int_{0}^{L} \frac{\operatorname{Corr}_{R_{1}} d}{T_{s_{1}} v} x d x=\frac{\operatorname{Corr}_{R_{1}}}{T_{s_{1}}} \frac{d}{2 v} L^{2} .
$$

We will use the above expression in the following sections.

\section{Continuous Box Stream, Two Readers}

We now add a second RFID reader to the scenario of section 5 and examine the case of a continuous stream of boxes moving past a pair of readers with completely overlapping fields. This is depicted in figure 4(b). A stream of boxes with tag density $d$ of boxes on the conveyor belt is passing through the interrogation zones of readers $R_{1}, R_{2}$. We wish to provide an optimal scheduling for the two readers. Equivalently, we wish ascertain at what points in time the readers should be switched.

Firstly, we use equation 6 to compute the number of tags read by reader $R_{2}$. We obtain

$$
R_{2} \text { read }=\frac{\operatorname{Corr}_{R_{2}}}{T_{s_{R_{2}}}} \frac{d}{2}\left(T_{1}-T_{0}\right)^{2} .
$$

Similarly, the number of tags Reader $R_{1}$ would have read had it been active during this initial period is given by

$$
R_{1} \text { read }=\frac{\text { Corr }_{R_{1}}}{T_{s_{R_{1}}}} \frac{d}{2}\left(T_{1}-T_{0}\right)^{2} .
$$

It immediately follows that one should only activate reader $R_{2}$ in the period $\left[T_{0} T_{1}\right]$ in one of the following two cases. Either

$$
\frac{\operatorname{Corr}_{R_{2}}}{T_{s_{R_{2}}}} \geq \frac{\operatorname{Corr}_{R_{1}}}{T_{s_{R_{1}}}}
$$

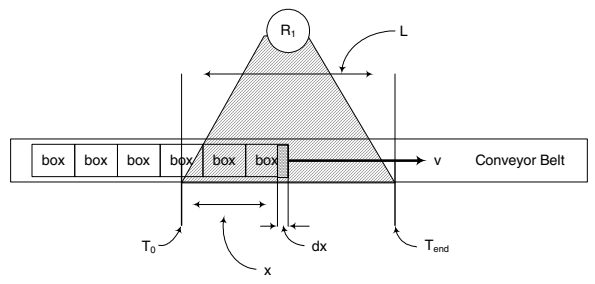

(a)

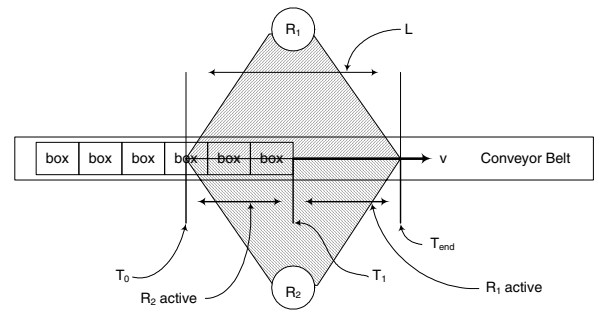

(b)

Fig. 4. a) The continuous box stream, one reader problem. b) The continuous box stream, two reader problem. 


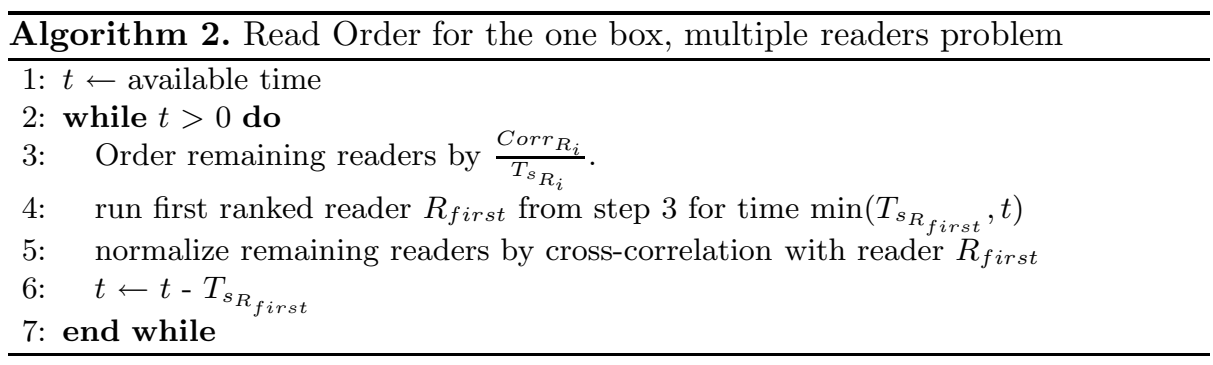

in which case

$$
T_{1}=\min \left(T_{s_{R_{2}}}, T_{\text {end }}\right),
$$

or reader $R_{1}$ achieves saturation, in which case

$$
T_{1}=\min \left(T_{0}, T_{\text {end }}-T_{s_{R_{1}}}\right) .
$$

This result is consistent with Algorithm 1, One first assigns all the available time to the fastest reader up to its saturation time. One then assigns any remaining time to the next fastest virtual reader. This algorithm can be applied recursively to scenarios with more than two readers as can be seen in algorithm 2 .

\section{Continuous Box Stream, Multiple Readers}

Let us now return to the RFID reader configuration from section 4, but replace the single box with the continuous stream of boxes used in the previous two sections.

At this point, the reader is encouraged to refer to Appendix (section 9) for some background material on the graphical analysis of the collision problem in RFID networks.

The principal difference between this scenario and the single box version arises from RFID reader collision. In the single box regime, turning off a reader did not necessarily result in a loss of information. As a result, it was reasonable to schedule only one reader for reads at any given time. However, such is not the case with this scenario. There are always tags underneath every reader. As a result we wish to have as many readers as possible reading in parallel. Algorithm 3 thus merges the algorithms proposed in sections 4 and 6.

In essence, Algorithm 3 uses graph theory to identify reader sets that can be treated as individual readers. This temporary reader set is then activated as if it were a single reader. Readers that do not complete reading are then returned to the reader pool for the next iteration of the algorithm.

\subsection{Aggregated Partition Read Rates}

Algorithm 3 requires computing the aggregated tag read rate for a set of RFID readers. We describe this computation below. 


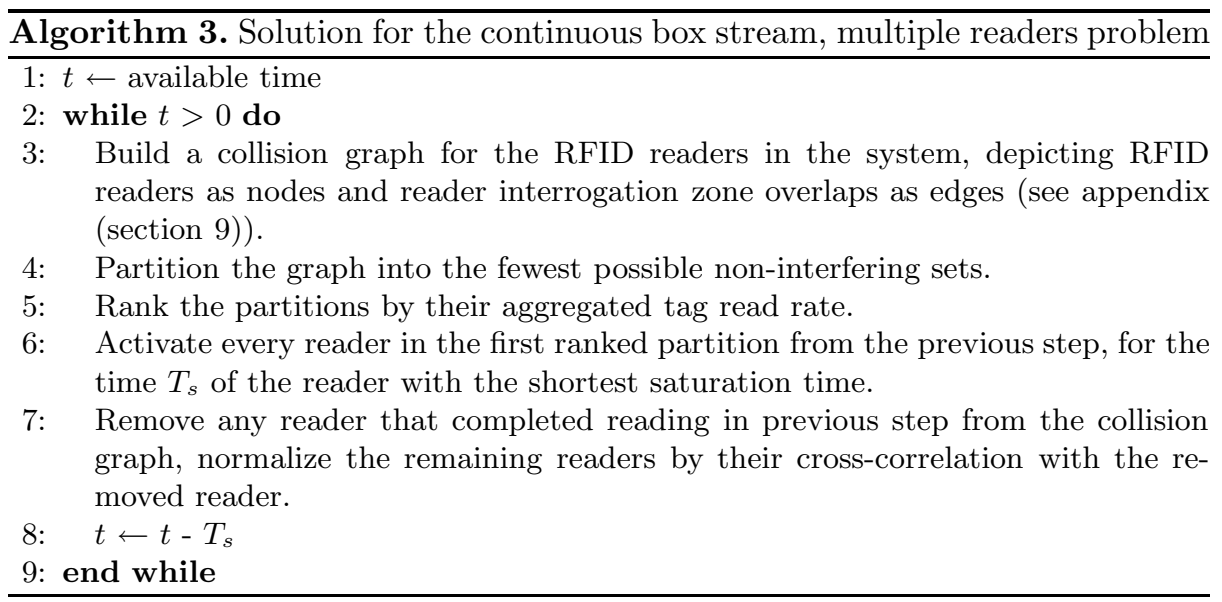

We intend for he aggregated correlation of a set of RFID readers to be, on the average, the fraction of unique tags read by the collection of readers from a collection of tags. With this in mind, we begin by computing the aggregated correlation Corr $_{\text {aggr }}$ for a pair of readers.

$$
\operatorname{Corr}_{\text {aggr. }}=\operatorname{Corr}_{R_{1}}+\operatorname{Corr}_{R_{2}}-r_{12}
$$

The aggregated read rate is obtained by scaling this with the saturation time of the system of two readers.

$$
\text { ReadRate }_{\text {aggr. }}=\frac{\text { Corr }_{\text {aggr. }}}{\min \left(T_{S_{1}}, T_{S_{2}}\right)}
$$

The general expression for the aggregated correlation for a set of $n$ readers $\left\{R_{i}\right\}_{1 \leq i \leq n}$ is obtained by applying the principal of inclusion and exclusion.

$$
\operatorname{Corr}_{\text {aggr. }}=\sum_{i} \operatorname{corr}_{R_{i}}-\sum_{i<j} r_{i j}+\sum_{i<j<k} r_{i j k}-\ldots \pm r_{12 \ldots n}
$$

We remind the reader that $r_{i j k}$ is the fraction of tags read in common by readers $R_{i}, R_{j}$ and $R_{k}$ from a set of tags, and so on.

While conceptually simple to evaluate, the aggregate correlation does place additional requirements on the data that must be measured when characterizing a system. In particular, it is not sufficient to obtain the cross-correlation between every pair of readers with overlapping interrogation zones. One must, instead, obtain all $2,3, \ldots, n$ way cross-correlations in order to perform optimal scheduling of multiple reader RFID systems. This is intuitively satisfying.

\section{Scheduling Domains}

It is not uncommon for readers to be separated by more than their reading range on the same conveyor belt. Such a scenario is depicted in Figure 5. Should each 


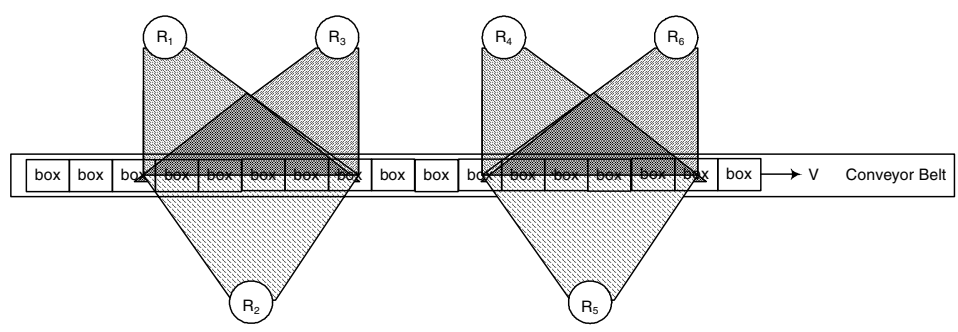

Fig. 5. Two multi-reader configurations on a conveyor belt

of these sets of readers be scheduled separately as independent "scheduling domains", or are there advantages to scheduling all of these seemingly independent readers as a single block?

We attempt to answer this question using the building blocks developed in the preceding sections to analyze the case of readers which fall into disjoint blocks with no overlaps between blocks.

Definition 3. A scheduling domain is a maximal set of RFID readers such that the collision graph of this topology is connected.

There are two scheduling domains in Figure 5. The first comprises readers $R_{1}, R_{2}$ and $R_{3}$, while the second consists of readers $R_{4}, R_{5}$ and $R_{6}$.

Now by the definition of a scheduling domain, readers in distinct scheduling domains do not physically interfere. However, there actually is an advantage to scheduling these readers together. The reason for this is cross domain correlation. While they are physically separate, their aggregated result is affected by the cross-correlation between each of the readers. Take the example of readers $R_{1}$ and $R_{4}$ in Figure 5. They do not physically interact. However, even if the event correlations of readers $R_{1}$ and $R_{4}$ with the set of tags are highest in their respective groups, if the cross-correlation of these two readers is very high, there may be an advantage to picking another reader in one of the groups in order to maximize the aggregated number of tags read by the readers.

\section{References}

1. V. Deolalikar, M. Mesarina, J. Recker, D. Das, and S. Pradhan. Perturbative time and frequency allocations for RFID reader networks, preprint 2005.

2. D. Engels, The Reader Collision Problem, Technical Report. MIT-AUTOID-WH007, 2001. http://www.autoidcenter.org/research/MIT-AUTOID-WH-007.pdf

3. F. Harary, Graph Theory, Addison-Wesley 1969.

4. I. Katzela and M. Naghshineh. Channel assignment schemes for cellular mobile telecommunication systems: A comprehensive survey. IEEE Personal Communications, pp. 1031, June 1996.

5. E. Malesinska. Graph-Theoretical Models for Frequency Assignment Problems. PhD thesis, Technischen Universitt Berlin, 1997. 
6. K. Yao, R. Hudson, C. Reed, D. Chen, and F. Lorenzelli. Blind Beamforming on a Randomly Distributed Sensor Array System. IEEE Journal on Selected Areas in Communications, Vol. 16, No. 8, October 1998.

7. F. Zhao, J. Shin, and J. Reich, "Information-Driven Dynamic Sensor Collaboration", IEEE Signal Processing Magazine, March 2002.

\section{Appendix: The Collision Graph of a RFID Network}

We provide below some elementary facts about the collision graph of a RFID network. For a more detailed graphical analysis of the collision problem for RFID networks, the reader is referred to [1] [2].

An (undirected) graph $G$ is an ordered pair $G=(V, E)$ where $V$ is a set of vertices or nodes and $E$ is a set of unordered pairs of distinct vertices, called edges. A subset of $V$ is called an independent set if there are no edges between vertices in $V$. An independent set is said to be maximal if the addition of any more vertices will result in a set that is not independent.

A simple undirected graph $G=(V, E)$ is called bipartite if there exists a partition of the vertex set $V=V_{1} \cup V_{2}$ where both $V_{1}$ and $V_{2}$ are independent

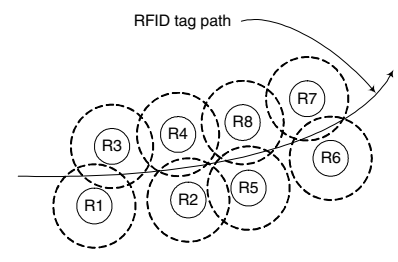

(a)

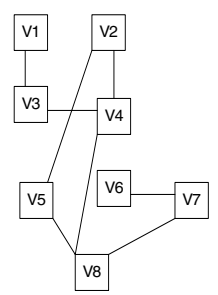

(b)

Fig. 6. RFID Reader partition example. a) RFID reader sample layout. b) Collision graph. Vertices represent the respective RFID reader, edges represent a collision, if both readers are on.

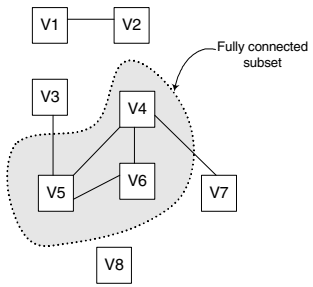

(a)

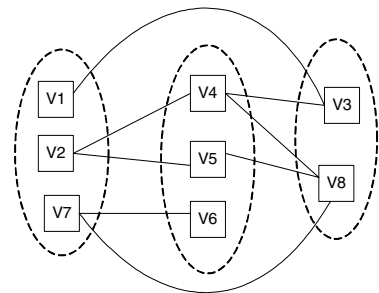

(b)

Fig. 7. a) The co-graph of figure 6(b), where each edge represents no collision. b) Graph partitioning into independent subsets. 
sets. In general, a graph is called $n$-partite when its vertex set $V$ can be written as $V=V_{1} \cup V_{2} \ldots \cup V_{n}$, where $V_{1}, V_{2}, \ldots, V_{n}$ are all independent sets.

Given a collection of RFID readers laid out in some manner, we can construct the associated collision graph $G=(V, E)$ where each vertex $v \in V$ corresponds to a RFID reader and each edge $e \in E$ represents a potential reader field collision between two readers. For example, the collision graph corresponding to the RFID reader layout of Figure 6(a) is given in Figure 6(b). Furthermore, the graph partitioned into independent sets of non-colliding readers as in Figure 7 (b).

Readers in any given partition of the collision graph can read simultaneously without interference. Unlike the "one box" problem, there will always be boxes in range every reader, thus it makes sense to fire every reader in a partition when firing one reader in the partition. 